\title{
Journal of NeuroEngineering and

\section{Effects of an adapted physical activity program in a group of elderly subjects with flexed posture: clinical and instrumental assessment Maria Grazia Benedetti*, Lisa Berti, Chiara Presti, Antonio Frizziero and Sandro Giannini}

Address: Movement Analysis Laboratory, Rizzoli Orthopaedic Institute, via di Barbiano 1/10, 40136 Bologna, Italy

Email: Maria Grazia Benedetti* - benedetti@ior.it; Lisa Berti - lisa.berti@ior.it; Chiara Presti - claire.press@gmail.com; Antonio Frizziero - segreteria.lanmov@ior.it; Sandro Giannini - giannini@ior.it

* Corresponding author

Published: 25 November 2008

Journal of NeuroEngineering and Rehabilitation 2008, 5:32 doi:10.1 186/1743-0003-5-32

This article is available from: http://www.jneuroengrehab.com/content/5/I/32

(c) 2008 Benedetti et al; licensee BioMed Central Ltd.

This is an Open Access article distributed under the terms of the Creative Commons Attribution License (http://creativecommons.org/licenses/by/2.0), which permits unrestricted use, distribution, and reproduction in any medium, provided the original work is properly cited.
Received: 20 February 2008

Accepted: 25 November 2008

\begin{abstract}
Background: Flexed posture commonly increases with age and is related to musculoskeletal impairment and reduced physical performance. The purpose of this clinical study was to systematically compare the effects of a physical activity program that specifically address the flexed posture that marks a certain percentage of elderly individuals with a non specific exercise program for 3 months.
\end{abstract}

Methods: Participants were randomly divided into two groups: one followed an Adapted Physical Activity program for flexed posture and the other one completed a non-specific physical activity protocol for the elderly. A multidimensional clinical assessment was performed at baseline and at 3 months including anthropometric data, clinical profile, measures of musculoskeletal impairment and disability. The instrumental assessment of posture was realized using a stereophotogrammetric system and a specific biomechanical model designed to describe the reciprocal position of the body segments on the sagittal plane in a upright posture.

Results: The Adapted Physical Activity program determined a significant improvement in several key parameters of the multidimensional assessment in comparison to the non-specific protocol: decreased occiput-to-wall distance, greater lower limb range of motion, better flexibility of pectoralis, hamstrings and hip flexor muscles, increased spine extensor muscles strength. Stereophotogrammetric analysis confirmed a reduced protrusion of the head and revealed a reduction in compensative postural adaptations to flexed posture characterized by knee flexion and ankle dorsiflexion in the participants of the specific program.

Conclusion: The Adapted Physical Activity program for flexed posture significantly improved postural alignment and musculoskeletal impairment of the elderly. The stereophotogrammetric evaluation of posture was useful to measure the global postural alignment and especially to analyse the possible compensatory strategies at lower limbs in flexed posture. 


\section{Background}

The aging process modifies normal postural alignment, and flexed posture commonly increases with age. Thoracic kyphosis and protrusion of the head, and in more severe cases, knee flexion, characterize flexed posture [1-5].

Although the precise etiology of flexed posture (FP) is unknown, its pathophysiology in the elderly is most likely multifactorial and can be associated with low bone mineral density and consequently vertebral fractures, and also degenerative alterations of intervertebral disks [2,6-12]. It is also related to musculoskeletal and neuromuscular impairments [13-15]: deficit in spinal extensor muscle strength and shoulder and hip range of motion decreasing have been correlated with flexed posture [1,14-18]. Increased flexed posture has been associated with less independence when performing activities of daily living and reduced physical performance, such as impaired balance, reduced postural control and slower walking, as well as risk of falling $[1,13,14,19-26]$. Moreover kyphosis and compensative cervical and lumbar spine hyperlordosis can cause pain due to ligaments and muscles impairment $[11,22]$.

There is no standard approach to measure flexed posture and classification methods are very complex [27]. Clinical assessments present the advantages of simplicity, low-cost and wide application, such as the occiput-to-wall distance [1] that classifies the severity of flexed posture. On the other hand, instrumental evaluation enables quantitative analysis of the global body alignment in flexed posture patients and especially the compensatory axial alterations at the head and lower limbs. In the literature, several studies have reported the use of goniometers, inclinometers [28,29], electrogoniometers [30], stadiometers [31-33] and photometric techniques [29,34-36]. The more recent stereophotogrammetric systems [37-39] seem to realize more reliable and valid evaluations of postural alignment analysis.

A sedentary life style is supposed to play a fundamental role in developing a flexed posture and there is evidence in literature that appropriate physical activity programs can correct this attitude [1].

There are few studies that investigate methods to improve flexed posture, and especially the relationship with the multiple impairments associated to flexed posture $[17,40,41]$. Sinaki $[14,16,42-47]$ focused most research on the correlation among osteoporosis, kyphosis and back extensors strength and defined an exercise program based on isometric back-extensor strengthening and proprioceptive postural retraining to contain flexed posture [17,48-50]. In different studies, the same author analyzed the role of the physical activity program in improving bal- ance, gait and quality of life and the consequences in reducing back pain, risk of falls and vertebral fractures $[17,46,49-51]$. The efficacy of these protocols seems to correlate to exercise specificity, but we failed to find sufficient randomized controlled trials in the literature that compare different programs.

Based on Sinaki's experience, we hypothesized that an Adapted Physical Activity (APA) program with specific exercises for flexed posture would improve postural alignment and physical performance in a more effective way than a non-specific physical activity protocol for the elderly. The purpose of this clinical study was to systematically compare the effects of a physical activity program that specifically address the flexed posture that marks a certain percentage of elderly individuals with a non specific exercise program for 3 months. A clinical multidimensional assessment [1], including evaluation of musculoskeletal impairment, motor function, and disability, and an instrumental assessment of global postural alignment were used as measures of outcome.

\section{Methods Subjects}

The study included elderly subjects aged over 65 years with flexed posture. Fifty-one participants were recruited from a Senior Club and provided written informed consent to take part in the study. After multidimensional clinical assessment seventeen subjects were not enrolled because they had one of the following exclusion criteria: central nervous system disorders, secondary osteoporosis, postural hypotension, disabling blindness or deafness, known malignant neoplasia, history of known vertebral fractures, obesity with Body Mass Index (BMI) >30, MiniMental State Examination (MMS) [52] >23, New York Heart Association (NYHA) classification $>1$, Short Physical Performance Battery $(\mathrm{SPPB})[53,54]$ with 1 item $=1$.

The 34 subjects included in the study, (28 women and 6 men) with a mean age of 70.9 years (S.D. 5.1), were randomly divided into two therapeutic groups: Group APA followed an Adapted Physical Activity (APA) program with specific exercises for flexed posture and Group NSPA followed a non-specific physical activity (NSPA) protocol for the elderly. In both groups exercises were performed 2 days a week for 1 hour and the program lasted for 3 months.

The participants that completed the two programs (at least $80 \%$ of sessions) were: 15 subjects in Group APA (12 females and 3 males) with a mean age of 71.5 (S.D. 4.3), weight $66.5 \mathrm{~kg}$ (S.D. 9.8), height $156.9 \mathrm{~cm}$ (S.D. 10.5) and BMI 27.22 (S.D. 4.5); and 13 subjects in Group NSPA (10 females and 3 males) with a mean age of 71.5 (S.D. 4.9), 
weight $72.7 \mathrm{~kg}$ (S.D. 11.1), height 159.5 (S.D. 8.7) and BMI 28.79 (S.D. 5.2).

The research protocol was approved by the Rizzoli Orthopedic Institute Ethics Committee.

\section{Intervention}

The exercises proposed for the APA group were aimed at improving flexibility at pelvic and shoulder girdle, and at strengthening back extensor muscles fighting the attitude to flexed posture. Most of exercises for back strengthening were selected among those proposed by Sinaki $[49,51,55-$ 57]. The set of exercises was discussed with the two physical trainers in charge for the groups management, in order to select exercises focused on specific impairment. We know in fact that both a deficit in spinal extensor muscle strength and reduction of flexibility at shoulders and hips have been correlated with flexed posture.

In both groups exercise sessions began with 10 minutes' warm-up and ended with 10 minutes' cool-down.

1. In a sitting position with hands behind the head, deepbreathing-in exercise combined with pushing elbows backwards. Then back to the initial position (10 repetitions).

2. In a sitting position with slightly flexed elbows, deepbreathing-in exercise combined with shoulder extension and adduction, and neck extension. Then back to the initial position (10 repetitions).

3. In a sitting position with arms along the sides, deepbreathing-in exercise combined with shoulder elevation. Then back to the initial position (10 repetitions).

4. In a sitting position with hands on thighs, deep-breathing-in exercise combined with shoulder abduction rotating palms upwards (10 repetitions).

5. In a sitting position holding a stick in two hands, deepbreathing-in exercise combined with raising the stick (8 repetitions).

6. In a sitting position with arms along the sides, lateral bending of the trunk while trying to touch the floor with fingers from one side to the other (8 repetitions).

7. In a standing position in front of a wall, arms overhead wall slides combined with neck extension (8 repetitions).

8. In a standing position with back touching the wall, starting from $90^{\circ}$ shoulders abduction and $90^{\circ}$ elbows flexion, complete shoulder abduction and elbow extension bringing hands over head (8 repetitions).
9. In a standing position with forearms on table, alternate hip extension (10 repetitions).

10. Supine with hip and knee flexion, and feet on the floor, anterior pelvic tilt while strengthening abdominal and glutei muscles (10 repetitions).

The non-specific physical activity protocol for the elderly adopted in Group NSPA consisted of global posture exercises through a floor training with the use of exercise balls for increasing joint mobility, muscle strength and flexibility.

\section{Clinical assessment}

A multidimensional clinical assessment [1] on each subject was performed, including anthropometric data (height, weight and BMI), clinical profile, and measures of musculoskeletal impairment, motor function, and disability.

The Comorbidity Severity Index of the Cumulative Illness Rating Scale [58] was adopted to evaluate physical health status. The level of pain was measured using visual-analog scales [59] in: neck, thoracic spine and lumbar spine. We used: the Mini-Mental State Examination (MMS) [52] for cognitive status, the Geriatric Depression Scale (GDS) [60] for evaluating depression and the Multidimensional Fatigue Inventory (MFI) [61] for measuring fatigue.

Goniometry measurements were used to record range of motion (ROM) in the hips, knees, and ankles bilaterally, obtaining the following data: hip flexion, hip extension, hip adduction, hip abduction, hip internal rotation, hip external rotation, knee flexion, knee extension, ankle plantarflexion, and ankle dorsiflexion.

Muscular strength was measured by means of manual muscle testing (MMT) [62] in the following groups of muscles: spine extensors, abdominal muscles, abductors, adductors, extensors and flexors of the hip, flexors and extensors of the knee, and dorsiflexors and plantarflexors of the ankle.

We used four previously reported tests [1] to evaluate lengthening capacity of pectoralis major, back extensors, hamstring muscles, and hip flexors. Motor function was explored by means of the Short Physical Performance Battery (SPPB) $[53,54]$ including balance test, gait speed test and chair-stand test. Disability was assessed using selfreport instruments: the Barthel Index [63] and the Nottingham Extended Activities of Daily Living (ADL) Index [64].

The clinical evaluation of flexed posture was performed by measuring the occiput-to-wall distance [1], while subjects 
stood with heels and back touching a wall. All the clinical evaluations were performed by the same blinded physiatrist for the physical activity program assignment.

\section{Instrumental assessment}

The instrumental assessment of posture was realized using a stereophotogrammetric system VICON 612 (Vicon Motion Systems, Oxford, UK) with 8 cameras (resolution 1.3 Megapixel, $100 \mathrm{~Hz}$ ).

Twenty-seven reflective markers were placed on the subjects at the following anatomical landmarks of head, trunk, pelvis, thigh, shank, foot:

Head: glabella, right temporomandibular joint, left temporomandibular joint

Trunk: right acromion, left acromion, spinous process of $7^{\text {th }}$ cervical vertebrae $(\mathrm{C} 7)$, medial point between the two spines of the scapula

Pelvis: right anterior superior iliac spine, left anterior superior iliac spine, right posterior superior iliac spine, left posterior superior iliac spine

Thigh: right greater trochanter, left greater trochanter, right lateral epicondyle, left lateral epicondyle

Shank: right tibial tuberosity, left tibial tuberosity, right head of the fibula, left head of the fibula, right lateral malleolus, left lateral malleolus

Foot: right calcaneus, left calcaneus, right first metatarsal head, left first metatarsal head, right fifth metatarsal head, left fifth metatarsal head.

During posture analysis, in order to relate the displacement of the marker arrays to the position of the 3D underlying bones, the Total 3D Gait was used [65]. The protocol developed for kinematic analysis of posture was designed at Rizzoli Orthopedic Institute and based on the Cast Protocol $[65,66]$. For the anatomical reconstruction of body segment motion, the following anatomical landmarks were calibrated: occipital protuberance, spinous process of $5^{\text {th }}$ lumbar vertebrae (L5), right medial epicondyle, left medial epicondyle, right medial malleolus, left medial malleolus, right second metatarsal head, and left second metatarsal head.

The subjects stood in an upright position, with arms crossed and feet parallel (Fig. 1). For each subject 3 posture assessments, of 10 seconds' duration, were performed. For the first registration, the subjects were asked to stare at a fixed point one meter from away, at eye level (static) (Fig. 1A). For the second registration the partici- pants were asked to stare at a visual target 30\% higher (extension) (Fig. 1B) than their eye level. For the third registration the participants were asked to stare at a visual target 30\% lower (flexion) (Fig. 1C) than their eye level. Three repetitions for each condition were registered for each subject.

A specific posture model was constructed to describe the reciprocal position of the body segment with 8 angles on the sagittal plane (Fig. 2). These angles were defined as follows:

- head protrusion: the supplementary angle to the angle between head and trunk

- trunk flexion: the supplementary angle to the angle between trunk and pelvis

- right and left hip flexion: the supplementary angle to the angle between pelvis and femur

- right and left knee flexion: the supplementary angle to the angle between femur and shank

- right and left ankle dorsiflexion: the angle between shank and foot

\section{Statistical analysis}

Continuous data were summarised in terms of means and standard deviation of the mean. The differences between baseline and 3 months follow up were investigated by the paired T-Test when the variances were homogenous and the Wilcoxon test when the variances were not homogeneous. All the analysis were considered significant for $\mathrm{p}<$ 0.05. Statistical Analysis was carried out by SPSS 15.0.

\section{Results}

\section{Clinical assessment}

Comparing the multidimensional clinical assessment performed at baseline and at 3 months, we noticed a statistically significant improvement of the occiput-to-wall distance (Table 1) only in Group APA.

Furthermore, we observed a greater improvement in lower limb range of motion in Group APA compared to Group NSPA; in the first group, many parameters increased (hip extension, knee flexion and extension and ankle dorsiflexion), whereas in the second one only knee flexion and extension increased (Table 1).

Considering muscular lengthening capacity, Group APA showed an improvement in three of the four flexibility tests regarding pectoralis major, hamstrings and hip flexors. Conversely, in Group 2 only the hamstrings flexibility increased (Table 1). 

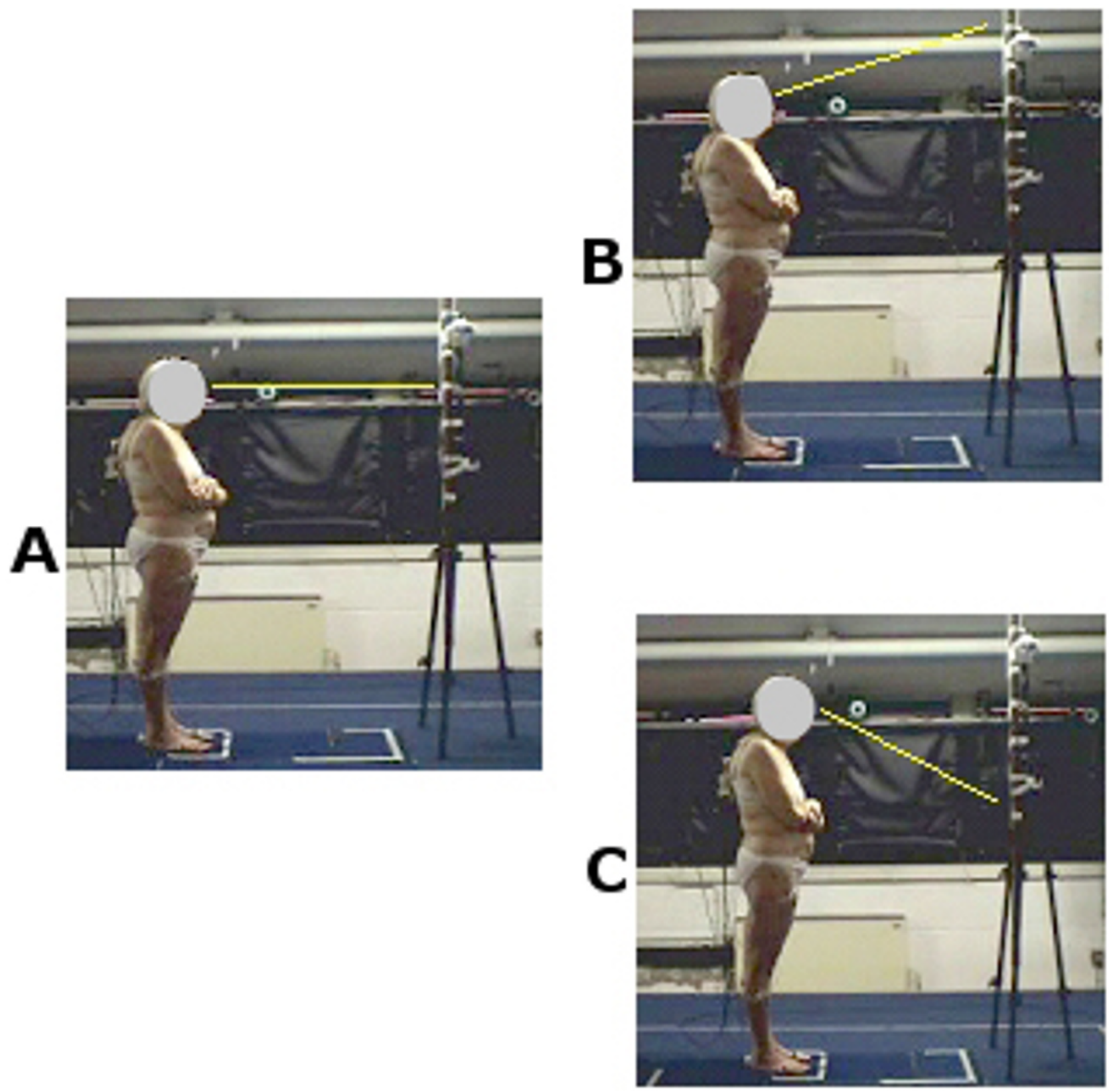

Figure I

Experimental set-up. The subjects stands in an upright position staring at a target at eye level (static) (fig. IA), 30\% higher (extension) (fig. IB) and 30\% lower (flexion) (fig. IC) than eye level.

In both groups improving in abdominal muscles strength was evident after the exercise programs, but spine extensors significantly increased their strength only in Group APA (Table 1).

Short Physical Performance Battery scores improved in both groups: in Group NSPA with a statistically significant difference, whereas the difference was close to significance $(\mathrm{p}=0.07)$ in Group APA (Table 2).
After exercise, there was no statistically significant difference in the two groups in the following scores: Mini-Mental State Examination, Geriatric Depression Scale, Multidimensional Fatigue Inventory, Barthel Index and Nottingham Extended Activities of Daily Living Index (Table 2).

The level of pain, evaluated by visual-analog scales, was only reduced in lumbar spine by both activity programs in a statistically significant way. 


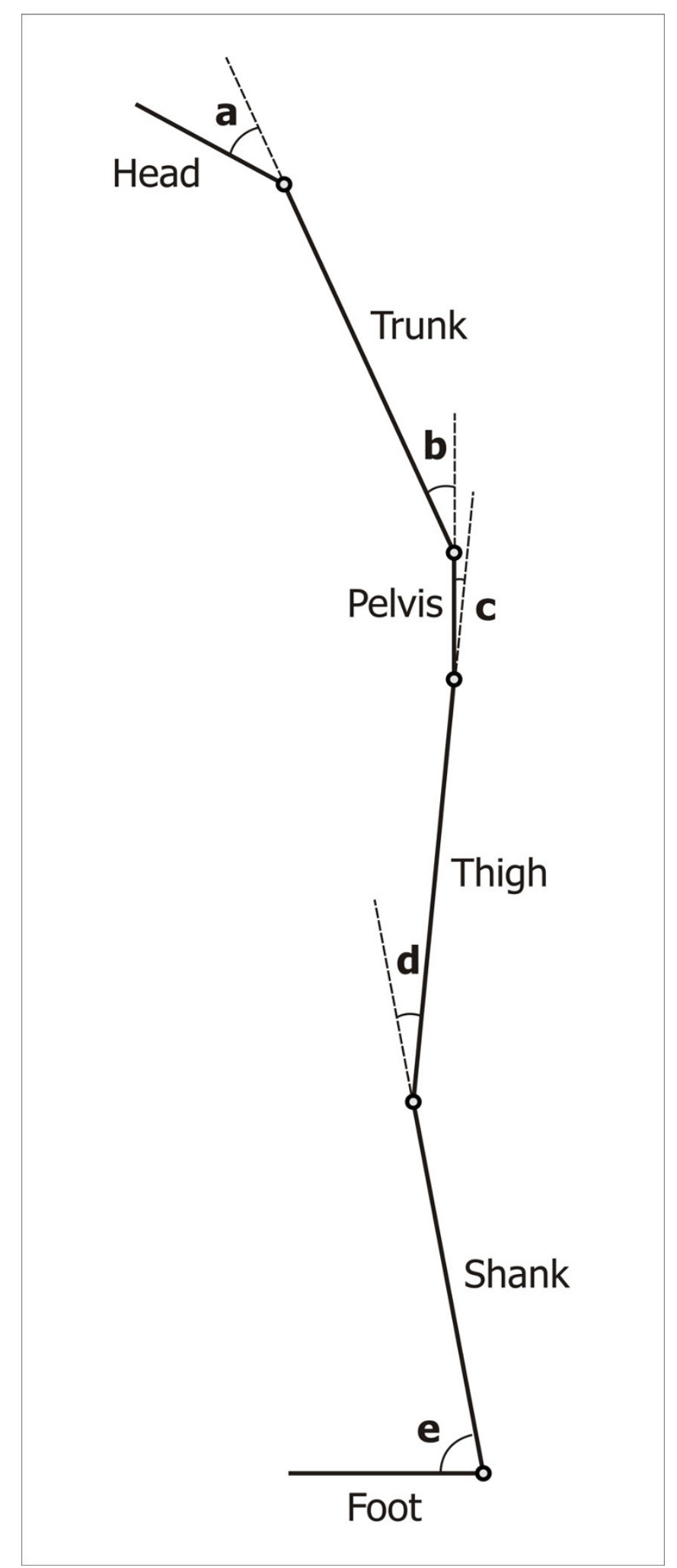

Figure 2

Postural model. The posture model describes the reciprocal position of the body segments with the following angles on the sagittal plane: head protrusion (a), trunk flexion (b), hip flexion (c), knee flexion (d), ankle dorsiflexion (e). 
Table I: Clinical assessment in Group APA (Adapted Physical Activity) and in Group NSPA (Non-Specific Physical Activity) at baseline and 3 months.

\begin{tabular}{|c|c|c|c|c|c|c|c|c|c|c|}
\hline & \multicolumn{4}{|c|}{ Group APA } & \multicolumn{6}{|c|}{ Group NSPA } \\
\hline & \multicolumn{2}{|c|}{ baseline } & \multicolumn{2}{|c|}{ after 3 months } & \multirow[t]{2}{*}{$\mathrm{P}$} & \multicolumn{2}{|c|}{ baseline } & \multicolumn{2}{|c|}{ after 3 months } & \multirow[t]{2}{*}{$\mathrm{P}$} \\
\hline & mean & SD & mean & SD & & mean & SD & Mean & SD & \\
\hline Occiput-to wall distance & 7.42 & 1.96 & 6.00 & 1.93 & 0.001 & 8.48 & 2.63 & 7.88 & 2.28 & ns \\
\hline \multicolumn{11}{|l|}{ Pain score } \\
\hline Lumbar spine & 4.97 & 2.43 & 2.73 & 2.66 & 0.01 & 4.83 & 2.40 & 1.85 & 1.95 & $* 0.003$ \\
\hline \multicolumn{11}{|l|}{ ROM } \\
\hline $\mathrm{R}$ hip extension & 12.60 & 5.32 & 20.40 & 7.13 & 0.002 & 14.62 & 7.40 & 16.31 & 4.07 & ns \\
\hline L hip extension & 12.20 & 5.59 & 20.07 & 6.66 & 0.003 & 13.23 & 7.47 & 16.15 & 3.71 & ns \\
\hline $\mathrm{R}$ knee flexion & 91.13 & 1.36 & 137.47 & 6.64 & $*_{0} 0.002$ & 98.54 & 1.29 & 136.00 & 5.35 & 0.008 \\
\hline L knee flexion & 92.27 & 3.83 & |38.07 & 4.83 & $* 0.003$ & 98.46 & 2.70 & $|37.3|$ & 5.10 & 0.009 \\
\hline R knee extension & 3.00 & 3.56 & 0.00 & 0.00 & $* 0.01$ & 2.85 & 4.56 & 0.00 & 0.00 & $* 0.04$ \\
\hline L knee extension & 3.27 & 3.32 & 0.00 & 0.00 & $* 0.008$ & 2.08 & 2.87 & 0.00 & 0.00 & $* 0.04$ \\
\hline $\mathrm{R}$ ankle dorsiflexion & 4.47 & 6.06 & 12.27 & 9.66 & $* 0.002$ & 6.69 & 4.75 & 8.46 & 2.25 & ns \\
\hline $\mathrm{L}$ ankle dorsiflexion & 4.53 & 8.09 & 9.53 & 4.88 & $*_{0} 0.012$ & 6.00 & 3.76 & 7.85 & 3.80 & ns \\
\hline \multicolumn{11}{|l|}{ Flexibility } \\
\hline $\mathrm{R}$ pectoralis major, $\mathrm{cm}$ & 9.26 & 4.30 & 7.40 & 3.28 & 0.018 & 10.36 & 8.46 & 8.76 & 5.64 & ns \\
\hline $\mathrm{L}$ pectoralis major, $\mathrm{cm}$ & 9.20 & 4.01 & 7.03 & 3,57 & 0.015 & 9.53 & 6.10 & 9.15 & 5.17 & ns \\
\hline $\mathrm{R}$ hamstrings, degrees & 70.13 & 12.31 & 78.20 & 12.09 & 0.017 & 70.54 & 11.23 & 77.00 & 14.52 & 0.04 \\
\hline $\mathrm{L}$ harmstrings, degrees & 68.07 & 12.77 & 78.87 & 13.45 & 0.011 & 70.31 & 10.62 & 78.54 & 8.02 & 0.052 \\
\hline $\mathrm{R}$ hip flexors, cm & 2.33 & 1.79 & 1.57 & 1.33 & 0.079 & 2.12 & 1.64 & 1.81 & 1.50 & ns \\
\hline L hip flexors, cm & 2.50 & 1.93 & 1.20 & 0.88 & 0.003 & 2.02 & 1.10 & 1.38 & 1.19 & ns \\
\hline \multicolumn{11}{|l|}{ Muscle strength } \\
\hline Spine extensors & 4.21 & 0.89 & 4.86 & 0.36 & 0.013 & 4.31 & 1.18 & 4.69 & 0.85 & ns \\
\hline Abdominals & 3.80 & 1.52 & 4.80 & 0.41 & $* 0.017$ & 3.54 & 1.45 & 4.62 & 0.65 & ns \\
\hline
\end{tabular}

$\mathrm{p}$ calculated with T-test, ${ }^{*} \mathrm{p}$ calculated with Wilcoxon test, ns = non-significant

Only parameters with a statistically significant difference between baseline and 3 months in almost one group were expressed.

\section{Instrumental assessment}

Regarding posture instrumental assessment, we compared the angles obtained at baseline and at 3 months.

In the standing position with visual target at eye level (static), we found, after exercise, a reduction in flexed posture characterized by diminishing protrusion of the head and ankle dorsiflexion in Group APA (Table 3). Conversely, Group NSPA did not show any statistically significant differences in posture angles after the activity program (Table 3).

In the standing position with visual target at a higher lever (extension), both groups had a decrease in knee flexion at follow up, although in Group APA this difference was more significant (Table 3 ).

In the standing position with visual target at a lower lever (flexion), Group APA showed a statistically significant reduction of protrusion of the head and a reduction of ankle dorsiflexion after exercise (Table 3).

\section{Discussion}

Considering the multifactorial pathophysiology of FP, we enrolled patients according to strict inclusion criteria to avoid confounding factors.

The multidimensional clinical assessment [1] was an excellent mean to characterize the elderly population because, as documented in literature [52-54,60,61,63,64], specific for studying the complex clinical profiles and impairments in the geriatric field.

Based on the well known concept that back extensors weakness is definitely recognized as a key element in the pathophysiology of FP $[1,14,67]$, exercised for strengthening of back extensor muscles were included in the APA protocol. Other exercises in the APA protocol were addressed to the stretching of muscles of hip and shoulder 
Table 2: Comprehensive geriatric assessment in Group APA (Adapted Physical Activity) and in Group NSPA (Non-Specific Physical Activity) at baseline and 3 months.

\begin{tabular}{|c|c|c|c|c|c|c|c|c|c|c|}
\hline \multicolumn{5}{|c|}{ Group APA } & \multicolumn{6}{|c|}{ Group NSPA } \\
\hline & \multicolumn{2}{|c|}{ baseline } & \multicolumn{2}{|c|}{ after 3 months } & \multirow[t]{2}{*}{$P$} & \multicolumn{2}{|c|}{ baseline } & \multicolumn{2}{|c|}{ After 3 months } & \multirow[t]{2}{*}{$P$} \\
\hline & mean & SD & mean & SD & & Mean & SD & mean & SD & \\
\hline Mini-Mental State Examination (max 30) & 28.93 & 1.90 & 28.93 & 1.79 & ns & 28.69 & 1.97 & 28.62 & 2.02 & ns \\
\hline Geriatric Depression Scale (0-30) & 5.33 & 4.73 & 4.40 & 5.22 & ns & 4.08 & 2.53 & 3.62 & 2.59 & ns \\
\hline Multidimensionall Fatigue Inventory $(20-100)$ & 38.73 & 13.78 & 34.53 & 15.39 & ns & 37.62 & 10.02 & 36.31 & 8.60 & ns \\
\hline Short Physical Performance Battery (max 12) & 10.33 & 1.17 & 11.07 & 0.79 & 0.07 & 9.38 & 1.04 & 10.46 & 1.61 & 0.028 \\
\hline Barthel Index (max 100) & 98.33 & 2.44 & 99.00 & 2.07 & ns & 98.85 & 2.19 & 100.00 & 0.00 & ns \\
\hline Nottingham Extended ADL Index (max 22) & 20.80 & 1.20 & 21.00 & 1.00 & ns & 20.92 & 1.18 & 21.00 & 1.15 & ns \\
\hline
\end{tabular}

P calculated with T-test, *p calculated with Wilcoxon test,, ns = non-significant

flexors. Besides a hip flexor static contracture, common in the elderly [68], we have to consider in fact that retraction of hip flexors and of anterior muscles of shoulders and neck is an expected consequence of flexed posture.

The results support our primary hypothesis that a Physical Activity program adapted to the specific impairment is more effective in improving flexed posture in the elderly than a non-specific protocol. This effect is evident when considering the spine extensor muscle strength, signifi- cantly increased only in the APA group. Moreover, even if both exercise programs had positive effects on knee ROM and hamstrings flexibility, the specific APA protocol was more effective in those districts mainly involved in flexed posture $[1,22,40,44]$, modifying ROM of hip joint and increasing the flexibility of hip flexor muscles and of the pectoralis major. The improvement of these measures only in the Group APA confirmed the specificity of the APA program in modifying soft tissue retraction associated to flexed posture.

Table 3: Instrumental assessment in Group APA (Adapted Physical Activity) and in Group NSPA (Non-Specific Physical Activity) at baseline and 3 months.

\begin{tabular}{|c|c|c|c|c|c|c|c|c|c|c|}
\hline \multicolumn{6}{|c|}{ Group APA } & \multicolumn{4}{|c|}{ Group NSPA } & \multirow{3}{*}{$P$} \\
\hline & \multicolumn{2}{|c|}{ baseline } & \multicolumn{2}{|c|}{ after 3 months } & \multirow[t]{2}{*}{$\mathrm{P}$} & \multicolumn{2}{|c|}{ baseline } & \multicolumn{2}{|c|}{ after 3 months } & \\
\hline & mean & SD & mean & SD & & mean & SD & mean & SD & \\
\hline \multicolumn{11}{|l|}{ STATIC } \\
\hline head protrusion & 40.31 & 8.79 & 35.16 & 9.60 & 0.04 & 39.81 & 10.85 & 40.51 & 9.29 & ns \\
\hline $\mathrm{R}$ ankle flexion & 80.74 & 3.42 & 83.11 & 3.85 & 0.01 & 80.21 & 4.78 & 80.97 & 5.23 & ns \\
\hline $\mathrm{L}$ ankle flexion & 80.4 & 4.21 & 83.81 & 3.94 & 0.01 & 81.65 & 5.26 & 83.23 & 5.05 & ns \\
\hline \multicolumn{11}{|l|}{ EXTENSION } \\
\hline$R$ knee flexion & 6.58 & 2.67 & 4.10 & 2.41 & $<0.0005$ & 5.85 & 3.55 & 3.27 & 1.95 & 0.01 \\
\hline L knee flexion & 6.41 & 3.61 & 3.87 & 1.92 & $<0.0005$ & 6.29 & 5.11 & 3.87 & 1.84 & 0.04 \\
\hline \multicolumn{11}{|l|}{ FLEXION } \\
\hline head protrusion & 50.71 & 4.86 & 40.08 & 7.02 & $<0.0005$ & 49.89 & 7.11 & 46.96 & 5.36 & ns \\
\hline $\mathrm{R}$ ankle flexion & 80.14 & 3.34 & 83.45 & 3.60 & $<0.0005$ & 80.86 & 5.48 & 81.36 & 4.52 & ns \\
\hline $\mathrm{L}$ ankle flexion & 81.94 & 3.86 & 84.00 & 3.60 & 0.04 & 82.16 & 3.59 & 82.85 & 5.13 & ns \\
\hline
\end{tabular}

$\mathrm{p}$ calculated with $\mathrm{T}$-test, $\mathrm{ns}=$ non-significant

Only parameters with a statistically significant difference between baseline and 3 months in almost one group were expressed. 
As a global measure of outcome in quantifying flexed posture, it is relevant that the occiput-to-wall distance, considered a specific indicator of severity of FP [1], showed a statistically significant decrease only in the APA Group.

The instrumental assessment of posture allowed us to measure the global postural alignment and especially to analyse the possible compensatory strategies to FP. The experimental set up was quite simply and easily reproducible. Considering the complexity of classification methods for posture in the literature [27], we designed a specific biomechanical model based on five different angles on the sagittal plane. These angles, clinically meaningful, (head protrusion, trunk flexion, hip flexion, knee flexion, ankle dorsiflexion) referred to precise compensatory axial deviations to kyphosis, that are important components of FP $[1,21]$. The three different visual tests (static, extension, and flexion) were performed to study the postural adaptations during eye-level modifications; this analysis allowed us to define the impact that the axial deformity of FP has on daily living activities (ADL), simulating dynamic activities which could be performed during ADL. While any compensatory movement is expected in normal people when looking at the top or down due to normal postural alignment and adjustment ability, we hypothesized that people with flexed posture and reduced mobility at the level of upper spine would adopt possible compensation strategies, mainly bending the knees and increasing ankle dorsiflexion in order to control balance.

Even in the instrumental evaluation, the specific APA program provided more significant improvements in the control of postural alignment than the non-specific protocol. In detail, both in static and flexion positions we observed a reduction in the flexion of the head and dorsiflexion of the ankle. In the extension position, the two programs determined the same positive effects with the decrease in knee flexion.

The more aligned head position instrumentally measured in the static posture and in the "extension test" in the APA Group confirms the reduction of the "occiput to wall distance" clinically measured. Moreover instrumental postural assessment highlights the increased ankle dorsiflexion as a compensatory strategy adopted by patients in order to maintain balance before the physical activity period, which reduces after training. The recovery of a better alignment of head and spine after specific exercise reduces the need of a biomechanical compensation at the ankle. The same mechanism can be supposed for the reduction of knee flexion in the "flexion test". In this case the improvement was observed in both groups, even more evident in the APA Group.
General physical performance increased in both groups, as the SPPB scores demonstrated. This was probably due to the positive effects that exercises, in general, have on balance, gait and motor function in the elderly. Conversely, after analyzing the Barthel Index [64] and the Nottingham Extended Activities of Daily Living Index [65] we could not find any improvement in the disability of the elderly after exercise. The reason for this can be attributed to the high-level of independence of the elderly population before treatment with a possible ceiling effect of score systems used. The slight association with disability might be due to the use of effective compensatory strategies even in the presence of severe FP, as previously hypothesized [1]. This finding confirms the results of other authors who found a weak correlation between severity of FP and disability [1,4,21]. The measure of cognitive status [52], depression [60], and fatigue [61] did not reveal any statistically significant changes; these results were consistent with the high values found before exercise. All patients complained of lumbar pain, improved after both the physical activity programs. As back pain is probably related to abnormal stress of muscles and ligaments $[11,22]$, it is reasonable that even a postural non-specific physical activity program can be effective in relieving this symptom.

The Adapted Physical Activity program was inspired by the well-documented Sinaki approach, based on the very important role of Spinal Proprioceptive Extension Exercise Dynamic (SPEED) program $[51,55,56]$. Sinaki amplified her own protocol with the use of a spinal weighted kypho-orthosis (WKO) to increase a patient's perception of spinal positioning $[17,43,50]$. We limited our program to the 10 selected exercises without using any orthosis.

The good results of the present trial are in agreement with findings of previous studies $[14,17,40,41,44,48]$ that investigated methods to improve flexed posture, all based on back-extension strengthening exercises. However, it was not possible to make a precise comparison of previous findings due to the different measurement systems adopted. The peculiarity of our research consisted of the randomized controlled clinical trial and the instrumental quantitative analysis of flexed posture. A limitation is however related to the small sample size of eligible subjects due to strict criteria of inclusion and different number of subjects which concluded the study in the two groups. The larger benefit achieved with the APA program compared to the non-specific activity program essentially refers to the investigation herein presented as a preliminary study. Further development of the present research is required particularly to calibrate the optimal amount of exercise to be administered taking into account even individual impairment. As Sinaki [46] affirmed, an exercise program aimed at maintaining muscular strength and 
flexibility is characterized by the principle of reversibility, so discontinuation reverses the improvement to pre-exercise levels. For this reason, the results of this research may be useful in developing a long-term Adapted Physical Activity program for the elderly aimed at containing and preventing FP.

\section{Conclusion}

The Physical Activity program adapted for people with flexed posture improved postural alignment and musculoskeletal impairment more effectively than a non-specific physical activity protocol. The increasing of back extensors strength, the increasing in the flexibility of pectoralis, hip flexors and hamstrings muscles correspond to the reduction of $\mathrm{FP}$, as measured by means of the occiput-wall distance. The instrumental assessment, based on a clinically oriented, reliable biomechanical model, allowed to measure the global postural alignment in patients with FP before and after physical activity trials and especially to analyse the possible compensatory strategies at the head and lower limbs.

\section{Competing interests}

The authors declare that they have no competing interests.

\section{Authors' contributions}

MGB made substantial contributions to conception and design of the study, analysis and interpretation of data and she was involved in drafting the manuscript and revising it critically for relevant intellectual content. LB performed all the measurements and was involved in drafting the manuscript. CP and AF participated in data acquisition and analysis. SG gave final approval of the version to be published.

\section{Acknowledgements}

This study was supported by grants from Italian Ministry of Health in the framework of the project "Prevention of vertebral fractures and postural misalignment in osteoporotic elderly". The authors wish to acknowledge Francesco Benvenuti, MD, for the scientific contribution to the design of the study, Roberto Piperno MD for the organizational support, the Santa Viola Senior Club direction staff and physical trainers for participation to the project, and Elettra Pignotti for the statistical elaboration of data.

\section{References}

I. Balzini L, Vannucchi L, Benvenuti F, Benucci M, Monni M, Cappozzo, Stanhope SJ: Clinical Characteristics of Flexed Posture in Elderly Women. JAGS 2003, 5 I (I 0): I-8.

2. Ensrud KE, Black DM, Harris F, Ettinger B, Cummings SR: Correlates of kyphosis in older women. The Fracture Intervention Trial Research Group. J Am Geriatr Soc 1997, 45(6):682-687.

3. Benvenuti F: Physiology of human balance. Adv Neurol 200I, 87:4l-5I.

4. Ensrud KE, Nevitt MC, Yunis C, Cauley JA, Seeley DG, Fox KM, Cummings SR: Correlates of impaired function in older women. J Am Geriatr Soc 1994, 42(5):48I-489.

5. Brocklehurst JC, Robertson D, James-Groom P: Skeletal deformities in the elderly and their effect on postural sway. J Am Geriatr Soc 1982, 30:534-8.
6. Huang MH, Barret-Connor E, Greendale GA, Kado DM: Hyperkyphotic posture and risk of future osteoporotic fractures: the Rancho Bernardo study. J Bone Miner Res 2006, 21:419-23.

7. Ettinger B, Black DM, Palermo L, Nevitt MC, Melnikoff S, Cummings $S R$ : Kyphosis in older women and its relation to back pain, disability and osteopenia: The study of osteoporotic fractures. Osteoporos Int 1994, 4:55-60.

8. De Smet AA, Robinson RG, Johnson BE, Lukert BP: Spinal compression fractures in osteoporotic women. Patterns and relationship to hyperkyphosis. Radiology 1988, 166:497-500.

9. Twomey L, Taylor J: Age changes in lumbar intervertebral discs. Acta Orthop Scand 1985, 56:496-499.

10. Buckwalter JA: Aging and degeneration of the human intervertebral disc. Spine 1995, 20(II): $1307-14$.

II. Finsen V: Osteoporosis and back pain among the elderly. Acto Med Scand 1988, 223(5):443-449.

12. Riggs BL, Melton LJ III: The worldwide problem of osteoporosis: insights afforded by epidemiology. Bone 1995, I7(Suppl 5):505S-5IIS

13. Hinman MR: Comparison of thoracic kyphosis and postural stiffness in younger and older women. Spine J 2004, 4:4I3-7.

14. Sinaki M, Itoi E, Rogers JW, Bergstralh EJ, Wahner HW: Correlation of back extensor strength with thoracic kyphosis and lumbar lordosis in estrogen deficient women. Am J Phys Med Rehabil 1996, 75:370-4.

15. Ferrucci L, Baldinelli S, Cavazzini C, Lauretani F, Corsi A, Bartali B, Cherubini A, Launer L, Guralnik JM: Neurological examination findings to predict limitation in mobility and falls in older persons without a history of neurological disease. Am J Med 2004, I I 6:807-I5.

16. Sinaki M, Itoi E, Wahner HW, Wollan P, Gelzcer R, Mullan BP, Collins $D A$, Hodgson SF: Stronger back muscles reduce the incidence of vertebral fractures: a prospective 10 year follow-up of postmenopausal woman. Bone 2002, 30(6):836-4I.

17. Itoi E, Sinaki M: Effect of back-strengthening exercise on posture in healthy women 49 to $\mathbf{6 5}$ years of age. Mayo Clin Proc 1994, 69 (I I): I054-9.

18. Norkin CC, Lavangie PK: Joint structure and function. A comprehensive analysis Philadelphia: F.A. Davis Company Woodhull-McNeal AP; 1992.

19. Ryan SD, Fried LP: The impact of kyphosis on daily functioning. J Am Geriatr Soc 1997, 45: 1479-86.

20. Lynn SG, Sinaki M, Westerlind KC: Balance characteristics of persons with osteoporosis. Arch Phys Med Rehabil 1997, 78(3):273-7.

21. O'Brien K, Culham E, Pickles B: Balance and skeletal alignment in a group of elderly female fallers and nonfallers. J Gerontol $A$ Biol Sci Med Sci 1997, 52(4):B22I-B226.

22. Ryan PJ, Blake G, Herd R, Fogelman I: A clinical profile of back pain and disability in patients with spinal osteoporosis. Bone 1994, 15(I):27-30.

23. Gold DT: The clinical impact of vertebral fractures: quality of life in women with osteoporosis. Bone 1996, 18(3):185-189.

24. Cunha U, Leduc M, Nayak US, Isaacs B: Why do old people stoop? Arch Gerontol Geriatr 1987, 6:363-9.

25. Leveille SG, Guralnik JM, Hochberg M, Hirsch R, Ferrucci L, Langlois $J$, Rantanen $\mathrm{T}$, Ling S: Low back pain and disability in older women: independent association with difficulty but not inability to perform daily activities. J Gerontol A Biol Sci Med Sci 1999 , 54(10):M487-93.

26. Schenkman M, Morey M, Kuchibhatla M: Spinal flexibility and balance control among community-dwelling adults with and without Parkinson's disease. J Gerontol A Biol Sci Med Sci 2000, 55(8):M44I-5.

27. Oi N, Tobimatsu Y, Iwaya T, Okada Y, Gushiken S, Kusano S, Yamamoto M, Takakura $Y$, Suyama T: Reliability and validity of classification of senile postural deformity in mass examination. Tohoku J Exp Med 2004, 202(2): 105- 12.

28. Seo A, Seo T, Kakehashi M, Yoshinaga F: Development of posture measuring apparatus using goniometer and inclinometer. Sangyo lgaku 1992, 34(3):216-24.

29. Gill K, Krag MH, Johnson GB, Haugh LD, Pope MH: Repeatability of four clinical methods for assessment of lumbar spinal motion. Spine 1988, 13(1):50-3. 
30. Boocock MG, Jackson JA, Burton AK, Tillotson KM: Continuous measurement of lumbar posture using flexible electrogoniometers. Ergonomics 1994, 37(I): I75-85.

31. Bridger RS, Wilkinson D, van Houweninge T: Hip joint mobility and spinal angles in standing and in different sitting posture. Hum Factors 1989, 3 I (2):229-4I.

32. Stothart JP, McGill SM: Stadiometry:on measurement technique to reduce variability in spine shrinkage measurement. Clin Biomech 2000, I 5(7):546-8.

33. Rodacki CL, Fowler NE, Rodacki AL, Birch K: Technical note: repeatability of measurement in determining stature in sitting and standing postures. Ergonomics 200I, 44(I 2):1076-85.

34. Dunk NM, Chung YY, Compton DS, Callaghan JP: The reliability of quantifying upright standing postures as a baseline diagnostic clinical tool. J Manipulative Physiol Ther 2004, 27(2):9l-6.

35. Dunk NM, Lalonde J, Callaghan JP: Implications for the use of postural analysis as a clinical diagnostic tool: reliability of quantifying upright standing spinal postures from photographic images. J Manipulative Physiol Ther 2005, 28(6):386-92.

36. McEvoy MP, Grimmer K: Reliability of upright posture measurements in primary school children. BBMC Musculoskelet Disord 2005, 6(35):.

37. McClure PW, Esola M, Schreirer R, Siegler S: Kinematic analysis of lumbar and hip motion while rising from a forward, flexed position in patients with and without a history of low back pain. Spine 1997, 22(5):552-8.

38. Cromwell RL, Aadland-Monahan TK, Nelson AT, Stern-Sylvestre SM, Seder B: Sagittal plane analysis of head, neck, and trunk kinematics and electromyographic activity during locomotion. J Orthop Sports Phys Ther 200I, 3 I (5):255-62.

39. Fowler NE, Rodacki AL, Rodacki CD: Changes in stature and spine kinematics during a loaded walking task. Gait Posture 2006, 23(2): |33-4|

40. Katzman WB, Sellmeyer DE, Stewart AL, Wanek L, Hamel KA: Changes in flexed posture, musculoskeletal impairments, and physical performance after group exercise in community-dwelling older women. Arch Phys Med Rehabil 2007, 88:192-9.

41. Greendale GA, McDivit A, Carpenter A, Seeger L, Huang MH: Yoga for women with hyperkyphosis: results of a pilot study. Am Public Health 2002, 92: 1611-4.

42. Sinaki M, McPhee MC, Hodgson SF, Merrit JM, Kenneth Offord P: Relationship between bone mineral density of spine and strength of back extensors in healthy postmenopausal women. Mayo Clin Proc 1986, 6 I(2): I I6-I22.

43. Sinaki M, Wahner HW, Offord KP, Hodgson SF: Efficacy of nonloading exercises in prevention of vertebral bone loss in postmenopausal women: a controlled trial. Majo Clin Proc 1989, 64(7):762-769.

44. Sinaki M: Relationship of muscle strength of back and upper extremity with level of physical activity in healthy women. Am J Phys Med Rehabil 1989, 68(3): I34-8.

45. Sinaki M, Khosla S, Limburg PJ, Rogers JW, Murtaugh PA: Muscle strength in osteoporotic versus normal women. Osteoporos Int 1993, 3(I):8-12.

46. Sinaki M: Musculoskeletal challenges of osteoporosis. Aging 1998, I 0(3):249-62

47. Sinaki M, Brey RH, Hughes CA, Larson DR, Kaufman KR: Balance disorder and increased risk of falls in osteoporosis and kyphosis: significance of kyphotic posture and muscle strength. Osteoporos Int 2005, I 6(8): 1004-10.

48. Hongo M, Itoi E, Sinaki M, Shimada Y, Miyakoshi N, Okada K: Effects of reducing resistance, repetitions, and frequency of backstrengthening exercise in healthy young women: a pilot study. Arch Phys Med Rehabil 2005, 86(7): I 299-303.

49. Sinaki M, Lynn SG: Reducing the risk of falls through proprioceptive dynamic posture training in osteoporotic women with kyphotic posturing: a randomized pilot study. Am J Phys Med Rehabil 2002, 8 I (4):24l-6.

50. Sinaki $M$ : The role of physical activity in bone health: a new hypothesis to reduce risk of vertebral fracture. Phys Med Rehabil Clin N Am 2007, I 8(3):593-608.

5I. Sinaki M, Brey RH, Hughes CA, Larson DR, Kaufman KR: Significant reduction in risk of falls and back pain in osteoporotickyphotic women through a Spinal Proprioceptive Extension
Exercise Dynamic (SPEED) program. Mayo Clin Proc 2005 80(7):849-55.

52. Folstein MF, Folstein SE, McHugh PR: "Mini-mental state". A practical method for grading the cognitive state ofpatients for the clinician. J Psychiatr Res 1975, I 2(3): 189-198.

53. Guralnik JM, Seeman TE, Tinetti ME, Nevitt MC, Berkman LF: Validation and use of performance measures of functioning in a non-disabled olderpopulation: MacArthur studies of successful aging. Aging 1994, 6(6):410-9.

54. Guralnik JM, Simonsick EM, Ferrucci L, Glynn RJ, Berkman LF, Blazer DG, Scherr PA, Wallace RB: A short physical performance battery assessing lower extremity function. Association with self-reported disability and prediction of mortality and nursing home admission. J Gerontol 1994, 49:M85-M94.

55. Sinaki M, Mikkelsen BA: Postmenopausal spinal osteoporosis: flexion versus extension exercises. Arch Phys Med Rehabil 1984, 65(10):593-6.

56. Sinaki $M$, Grubbs NC: Back strengthening exercises: quantitative evaluation of their efficacy for women aged 40 to 65 years. Arch Phys Med Rehabil 1989, 70(I): I6-20.

57. Sinaki M: Exercise and osteoporosis. Arch Phys Med Rehabil 1989 , 70(3):220-9.

58. Miller MD, Paradis CF, Houck PR, Mazumdar S, Stack JA, Rifai AH, Mulsant B, Reynolds CF 3rd: Rating chronic medical illness burden in geropsychiatric practice and research. Application of the Cumulative IIIness Rating Scale. Psychiatry Res 1992 , 41:237-248

59. Huskisson EC, Jones J, Scott PJ: Application of visual-analogue scales to the measurement of functional capacity. Rheumatol Rehabil 1976, I 5:185-187.

60. Yesavage JA, Brink TL, Rose TL, Lum O, Huang V, Adey M, Leirer VO Development and validation of a geriatric depression screening scale: A preliminary report. J Psychiatr Res 1982, I 7:37-49.

61. Smets EM, Garssen B, Bonke B, De Haes JC: The Multidimentional Fatigue Inventory(MFI) psychometric qualities of an instrument to assess fatigue. J Psychosom Res 1995, 39:315-325.

62. Daniels LCW: Muscle Testing Techniques of Manual Examination Philadelphia: W. B. Saunders; 1980.

63. Mahoney FL, Barthel DW: Functional evaluation. The Barthel Index. Md State Med J 1965, I 4:6 I-65.

64. Lincoln NB, Gladman JR: The Extended Activities of Daily Living scale: A further validation. Disabil Rehabil 1992, I 4:4|-43.

65. Leardini A, Sawacha Z, Paolini G, Ingrosso S, Nativo R, Benedetti MG: $A$ new anatomically based protocol for gait analysis in children. Gait Posture 2007, 26(4):560-7I.

66. Cappozzo A, Catani F, Croce UD, Leardini A: Position and orientation in space of bones during movement: anatomical frame definition and determination. Clin Biomech 1995, I0(4): $171-178$

67. Kalliomaki JL, Siltavuori L, Virtama P: Stature and aging. J Am Geriariatric Soc 1973, 2 1:504-506.

68. Kerrigan DC, Xenopoulos-Oddsson A, Sullivan MJ, Lelas JJ, Riley PO: Effect of a hip Flexor-Stretching Program on gait in the elderly. Arch Phys Med Rehabil 2003, 84: I-6.

Publish with Bio Med Central and every scientist can read your work free of charge

"BioMed Central will be the most significant development for disseminating the results of biomedical research in our lifetime. "

Sir Paul Nurse, Cancer Research UK

Your research papers will be:

- available free of charge to the entire biomedical community

- peer reviewed and published immediately upon acceptance

- cited in PubMed and archived on PubMed Central

- yours - you keep the copyright 